\section{Comment on: Prediction of retinopathy of prematurity using the screening algorithm WINROP in a Saudi cohort of preterm infants}

\section{To the Editor}

I highly appreciate this extensive study on Retinopathy of prematurity (ROP), from Saudi Arabia. ${ }^{1}$ I want to share my experience both in field and in the medical college associated hospitals in this country. There is shortage of ophthalmologists in general and pediatric ophthalmologist in particular especially in ministry of health hospitals. Case is on record where the doctor caring for the babies (triplet) one of them got ROP, initially examined by general ophthalmologist and proved blind latter and treating doctor got involved in medico legal battle because the parents complained against the doctor. Prevalence of prematurity in Saudi Arabia is common. Infants born before 37 weeks from the first day of the last menstrual period (LMP) are termed premature by World Health Organization (WHO). Extreme low birth weight (ELBW) is used to describe infants with birth weight less than 1000 gm. Very low birth weight (VLBW) describes infants less than 1500 gm. And low birth weight (LBW) describes infants less than $2500 \mathrm{gm}$. at birth. The etiology of preterm birth is multifactorial and involves complex interactions between fetal, placental, uterine and maternal factors. ${ }^{2}$ Early detection of retinopathy of prematurity (ROP) in preterm infants is critical, especially with advancements in neonatal care and improved survival rates. However, a balance should be found between not missing any ROP requiring treatment and minimizing workload, saving resources, and reducing unnecessary examinations to fragile neonates. Mgharbil et al reported that they used a revised version of ROP screening guidelines by the American Academy of Pediatrics. Neonates screened for ROP in the neonatal intensive care unit of our institution between January 2016 and November 2018 were included. Of 1393 live births, 155 babies met the inclusion criteria. ROP occurred in 60/155 (38.7\%) screened babies while sixteen devel $\neg$ oped threshold ROP. All 16 babies who required treatment had both a birth weight $\leq 1000 \mathrm{~g}$ and a gestational age of $\leq 30$ weeks. Using the screening recommendations of the Canadian Policy, more infants would have been screened without diagnosing a case of ROP of any stage, and no case of ROP requiring treatment would have been missed com $\neg$ pared to the AAP recommendations. ROP requiring treatment is a rare occurrence in pre $\neg$ mature infants with a gestational age $>30$ weeks and body weight $>1000 \mathrm{~g}$ at our institute. Nonetheless, this is not an attempt to alter national screening guidelines. A multicenter prospective study with an adequate sample size is needed to assess whether guidelines for ROP screening should be altered in this category of neonates. ${ }^{3}$ Alabbasi et $\mathrm{al}^{4}$ found the prevalence of preterm birth among Saudi women in Jeddah city of Saudi Arabia, was $13.7 \%$. It is estimated that world-wide some 15 million preterm babies are born annually, at a rate of approximately 1 in 10 babies. Waheeb \& Al-Shehri ${ }^{5}$ in their study observed that the incidence of ROP was $33.7 \%$ in Jeddah. Laser therapy was very effective for the treatment of this disease and preventing progression of $\mathrm{ROP}$. Al-Qahtani et $\mathrm{al}^{2}$ reported in their study that $\mathrm{ROP}$ is one of the major diseases affecting premature infants.

The screening criteria of ROP should be broadened at least to include those infants with gestational age between 32 and 35 weeks. Although blindness is well-known consequence of ROP, it has not been encountered in our study population. ${ }^{6}$ Retinopathy of prematurity (ROP) is a multifactorial vasoproliferative retinal disorder that increases in incidence with decreasing gestational age. Approximately $65 \%$ of infants with birth weight less than $1250 \mathrm{gm}$. And $80 \%$ of those with birth weight less than $1000 \mathrm{gm}$. Will develop some degree of ROP. ${ }^{7}$ It may be acute (early stages) or chronic (late stages). ROP includes all stages of the disease and its squeal. In more than $90 \%$ risk infants, the course is one of spontaneous arrest and regression with little or no residual effects or visual disability. Fewer than $10 \%$ of infants have progression toward severe disease with significant extra retinal vasoproliferation, detachment of the retina, impairment of vision and cicatrization. Recent research has focused on maintaining oxygen saturation level for severely premature infants at level sufficient low to minimize the risk of ROP and sufficiently high to optimize survival. Premature infants with ROP are at higher risk of developing Strabismus, Amblyopia and refractory errors than the average child. The risk of vision loss from ROP can be reduced by timely screening of premature infants by an ophthalmologist. Prevention of ROP ultimately depends on prevention of premature birth and its attendant problems. On 14 March 2019, his excellency, the minister of health Saudi Arabia, Dr. Tawfiq bin Fawzan Al-Rabiah launched the Retinopathy Of Prematurity (ROP) National Program. In its first phase, the program covers Neonatal intensive care units in MOH's hospitals across the Kingdom. It uses 21 Digital cameras to depict the 
fundus of the preemies eyes and send pictures to the Hospitals retinal consultants, who make medical report and recommendation for treatment and send them electronically to the hospital. ${ }^{8}$ In conclusion currently, no proven methods are available to prevent ROP. There is need to provide ophthalmologists in deficient areas in ministry of health hospitals. To arrange and to update the knowledge and skills about neonatal ophthalmic examination for adult ophthalmologists. To make best use of ROP national programme launched Last year (2019).Doctors handling preterm babies should fully explain about outcome of ROP to the parents to be on the safer side.

Ghulam Nabi Bugshan Hospital Jeddah, Kingdom of Saudi Arabia

\section{Reply from the Author}

I read the correspondence and found it to be a general opinion on ROP screening in general.

Lina H. Raffa

Department of Ophthalmology King Abdulaziz University Hospital Jeddah, Kingdom of Saudi Arabia

\section{References}

1. Raffa LH, Alessa SK, Alamri AS, Malaikah RH. Prediction of retinopathy of prematurity using the screening algorithm WINROP in a Saudi cohort of preterm infants. Saudi Med J 2020; 41: 622-627.

2. Olitsky SE, Marsh JD. Disorders of retina \& viterous. Nelson text book of pediatrics. 21st ed. Phelidelphia (PA): Elsevier; 2020. p. 3377-2279.

3. Mgharbil Hassan Raffa E. L. Alessa S. Alamrib. A. Screening premature infants for retinopathy of prematurity in a tertiary hospital in Saudi Arabia. Ann Saudi Med 2020; 40: 87-93.

4. Alabbasi.K.H. Kruger E and Tennent M. Maternal Variables as Potential Modifiable Risk Indicators of Preterm Labor in Jeddah, Saudi Arabia. J Preg Child Health 2015; 2: 166.

5. Waheeb $S$, Alshehri K. Incidence of retinopathy of prematurity at two tertiary centers in Jeddah, Saudi Arabia. Saudi J Ophthalmol 2016; 30: 109-112.

6. Al-Qahtani B, Al-Otaibi M, Alabdulajabbar K, Selayem NB, Alshehri W, Omair A, et al. Retinopathy of prematurity incidence and risk factors in a tertiary hospital in Riyadh, Saudi Arabia. Middle East Afr J Ophthalmol 2020; 26: 235-239.

7. Leeman K T. and Vanderveen D.K. Retinopathy of Prematurity. Cloherty and Starks manual of neonatal Care. 8th ed. Philadelphia (PA): Wolters Kluwer; 2017. p. 986-992.

8. Ministry of Health. National E-Health Strategy. [cited 2020]. Available from: https://www.moh.gov.sa/en/Ministry/nehs/ Pages/vision2030.aspx 